\title{
Comparison of an Optical-Digital Restoration Technique with Digital Methods for Microscopy Defocused Images
}

\author{
R. Ortiz-Sosa, L.R. Berriel-Valdos, J. F. Aguilar \\ Instituto Nacional de Astrofísica Óptica y Electrónica, Puebla, Mexico \\ rosalinda@inaoep.mx
}

\begin{abstract}
An optical-digital technique for restoring defocused images, and based on the quasi-point source concept, is introduced and compared with two conventional digital methods. The comparison is performed on the restoration of defocused microscopy images obtained with a conventional microscope but with three different numerical apertures. The effects of these techniques on the aberrated images are shown. We discuss and conclude about the advantages of our proposed technique.
\end{abstract}

Keywords: Optical-digital restoration technique, digital methods, microscopy defocused images.

\section{Introduction}

Every imaging optical system $(O S)$ has a characteristic intensity point spread function (ipsf) associated, this function depends on the illumination wavelength $(\lambda)$, the pupil function of $O S$ and the numerical aperture $(N A)$. In presence of aberrations of the $O S$, or with defocus, the ipsf detected out of focus will contain all this information, that is why the knowledge of the ipsf allows us to evaluate the quality of the $O S$, and its use is widely applied in image formation theory.

Since, the concept of point source is a mathematical abstraction, very useful for developing theoretical results, but the experimental realizations it is necessary to use a real point source. We propose to use an object with enough small dimensions that the $O S$ cannot resolve, this kind of object is commonly known as a quasi-point source (qps) [1-3].

The detected image of a qps is then an estimated of the ipsf, and can also be used for generating of out-of-focus ipsf's. This corresponds with image of a qps when is located at different distances of the object plane.

Once these images have been detected it is possible to obtain the corresponding optical transfer functions $\left(O T F^{\prime} s\right)$ associated with every defocused position. Then, a Wiener [4] filter can be constructed with those images. This is in order to realize a typical deconvolution technique, and that is why we call this an optical-digital technique.

In this work, a comparison of three different methods of restoration is presented. That which uses the qps and other two techniques using the digital (OTF's) [5-7]. 


\section{Theoretical Background}

In an aberration-free $O S$, the intensity distribution $(g(x, y))$ in the image plane is related with the intensity transmittance $(o(x, y))$ of the object and with the ipsf $(h(x, y))$ my means of the so called image formation equation in intensity

$$
g(x, y)=h(x, y) \otimes o(x, y),
$$

where $(x, y)$ are the spatial rectangular coordinates and $\otimes$ indicates the convolution operation.

In the Fourier domain the equation takes the form

$$
G(u, v)=H(u, v) O(u, v),
$$

where $G$ is image spectrum, $O$ represents the spectrum of the transmittance of the object, and $H$ is the OTF; $(u, v)$ are the coordinates in the frequency domain.

When the image of a defocused object is detected, an aberration is introduced this known as defocus and is denoted by the term $\omega_{2,0}$ of the aberrations polynomial [8]. This aberration can be represented in the pupil function of the $O S$ as

$$
P_{d}(\rho)= \begin{cases}\exp \left[i k \omega_{2,0}\left(\rho^{2}\right)\right] & \text { if } \rho \leq R \\ 0 & \text { if } \rho>R\end{cases}
$$

where $k=2 \pi / \lambda, \rho=\sqrt{u^{2}+v^{2}}$ and $R$ is the radius of the pupil. The relation of $\omega_{2,0}$ with the displacement along the $z$ axis of the object from the object plane $(\Delta z=0)$ is of the form [9]:

$$
\omega_{2,0}=\frac{1}{2} N A^{2} \Delta z
$$

being $N A$ the numerical aperture of the $O S$.

The ipsf for an object located out-of-focus which is denoted by $h_{d}$, is the square modulus of the inverse Fourier transform $\left(\mathfrak{J}^{-1}\right)$ of the aberrated pupil function [10]:

$$
h_{d}(x, y)=\left|\Im^{-1}\left\{P_{d}(u, v)\right\}\right|^{2} .
$$

Then, equation (1) containing the effects of defocus and including the noise $(n)$, which is introduced in the detection process, is expressed in the form

$$
g_{d}(x, y)=h_{d}(x, y) \otimes o(x, y)+n(x, y)
$$

This noise can be reduced by mean of an average over a sequence of images captured for the same defocus position $\Delta z$. The image formation equation in terms of this average image is

$$
\bar{g}_{d}(x, y) \approx h_{d}(x, y) \otimes o(x, y)
$$

and its spectrum is rewritten as 
Comparison of an optical-digital restoration technique with digital methods for microscopy ...

$$
\bar{G}_{d}(u, v) \approx H_{d}(u, v) O(u, v) .
$$

In the other hand, the average image $\left(\bar{q}_{d}\right)$ of a qps is expressed in the form

$$
\bar{q}_{d}(x, y)=h_{d}(x, y) \otimes \operatorname{circ}\left(\frac{\sqrt{x^{2}+y^{2}}}{R}\right) .
$$

Since $R$ is of very small dimensions, this means that the circ function can be approximated with a Delta function $\operatorname{circ}\left(\sqrt{x^{2}+y^{2}} / R\right) \approx \delta(x, y)$, i.e the image of the a $q p s$ is approximated to the $i p s f_{d}$ :

$$
\bar{q}_{d}(x, y) \approx h_{d}(x, y)
$$

and, in the Fourier domain equation (10) is expressed as

$$
\bar{Q}_{d}(u, v) \approx H_{d}(u, v) .
$$

When the Wiener filter is used, the mean square error in the comparison between the focused image and the restored one is minimum. For this reason we have decided to use this filter for obtaining the spectrum of the restored image [5, 7]:

$$
\widehat{G}(u, v)=\frac{\bar{G}_{d}(u, v) H_{d}^{*}(u, v)}{\left|H_{d}(u, v)\right|^{2}+S_{n}(u, v) / S_{f}(u, v)^{\prime}}
$$

where $S_{n}$ corresponds to the power spectrum of the noise and $S_{f}$ is the power spectrum of the focused image; symbol * indicates conjugated complex.

Usually, in image restoration, when the values of the quotient in denominator of equation (12) this value is adjusted in an heuristically way for obtaining a good quality restoration we denote this factor with an $\varepsilon$, and selected the value of $\varepsilon=3 \times 10^{-3}$.

Replacing equation (11) in equation (12) for the Wiener filter we have:

$$
\hat{G}(u, v)=\frac{\bar{G}_{d}(u, v) \bar{Q}_{d}^{*}(u, v)}{\left|\bar{Q}_{d}(u, v)\right|^{2}+\varepsilon}
$$

and, finally, the inverse Fourier transform of equation (13) is an estimated of the image of the focused object.

\section{Digital Methods}

In the method proposed here, approximated of the defocused ipsf's of the $O S$ are obtained. However, other authors have used ipsf's numerically simulated $[5,6]$ and to obtain them, the equation (3) is used, where the defocus term $\omega_{2,0}$ of the aberration polynomial is introduced. The defocus amount is calculated according with the equation (4), from the a priori knowledge of the distance from the object plane $\Delta z=0$ at the moment in which the image is captured. 
Another method with our technique is compared is the so called Blind Deconvolution Algorithm. This algorithm is used when the amount of defocus in the captured image is unknown; in this case the ipsf is estimated by means of an iterative process. This algorithm uses the standard of Maximum Likelihood [7].

\section{Experiment}

The OS is a bright field Olympus microscope model BX51, used with three objectives, $4 \mathrm{X}(0.1), 10 \mathrm{x}(0.3)$ y $20 \mathrm{x}(0.5)$. The image sequences used in the experiment were captured with a monochromatic $C C D$ with a dynamic range of $0-255$. The $C C D$ has a $2448 \mathrm{H}$ X $2050 \mathrm{~V}$ pixels, and a pixel pitch of $3.45 \mu \mathrm{m}$. This Sony model XCL-5000. For digitizing the detected images a frame-grabber PCI-142924 from National Instruments.

The images introduced in our computing code were a segment of the originally captured of $512 \times 512$ pixels.

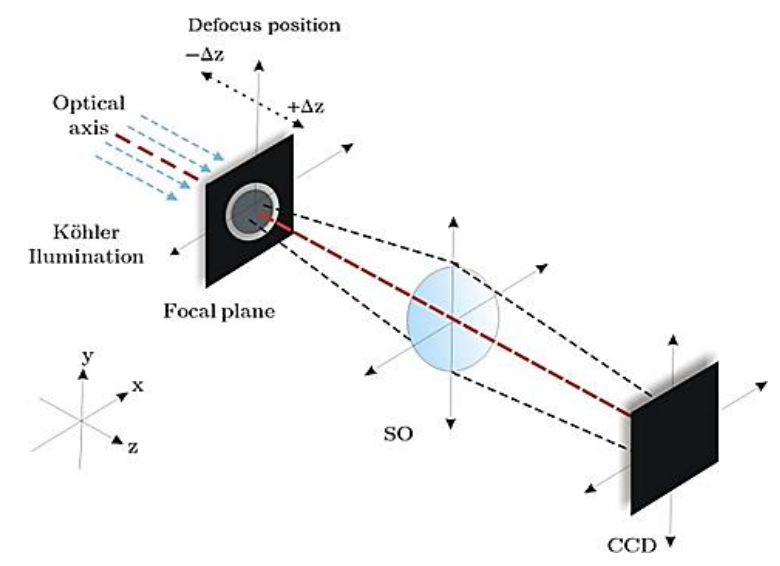

Fig. 1. Schematic diagram or the optical system.

For reducing the noise and other unwanted effects generated during the detection process, a series of images were averaged for each defocused position.

Considering that we wanted to keep a minimum capturing time, and after several proofs for determining the minimum variance in which the noise is reduced, a number of 20 images was selected for each defocus position.

For the generation of the $\bar{q}_{d}$ a pinhole of $1 \pm 0.5 \mu \mathrm{m}$ was used. This was previously verified that fulfills the requirements to be considered as a $q p s$ for the $O S$ used in this work.

As the object we used a typical resolution test target. A region of the object containing high frequencies was analyzed.

\section{$5 \quad$ Results}

We show the results obtained with our optical-digital technique, and also those obtained with the other two digital methods. 


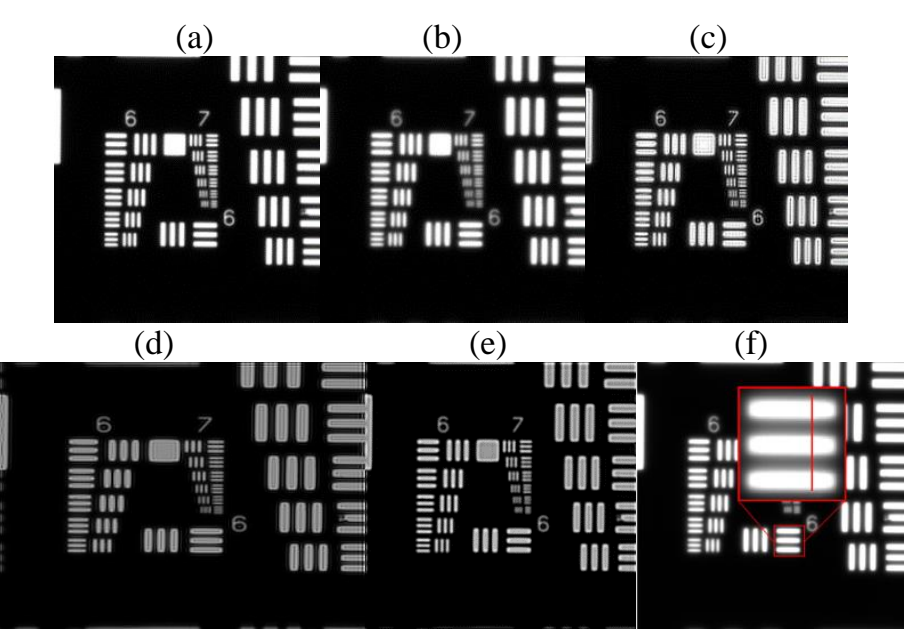

Fig. 2. a) in-focus image of the resolution test target detected with a microscopy objective $4 \mathrm{X}(0.1) ; \mathrm{b})$ defocused image of the object which amount of defocus is $\omega_{2,0}=0.65 ; \mathrm{c}$ ) restoration of the defocused using a simulated ipsf; d) restoration with the Blind deconvolution algorithm, with 100 iterations; e) restoration with our proposed optical-digital technique; f) this image is showing the transverse section that is used for the profiles plotted in the figure 3.

The results are presented in three groups, one for each objective lens used in the experiment. The first group is shown in Figure 2, it corresponds to the objective $4 \mathrm{X}(0.1)$ this includes the restoration obtained with our proposed method and also the restorations obtained with the other two methods. In the same way, the group in Figure 4 is for the images obtained with the objective $10 \mathrm{X}(0.3)$ and the group in Figure 6 is for the objective $20 \mathrm{X}(0.5)$.

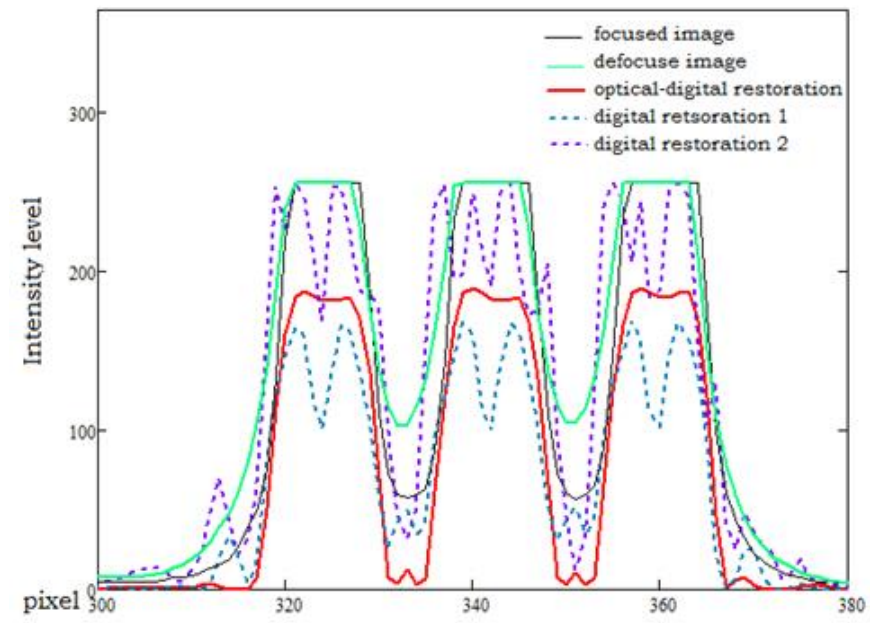

Fig. 3. Intensity profiles in the indicated region in Figure 2(f). Optical-digital technique (continuous red line), using a simulated ipsf (dotted blue line), the Blind deconvolution algorithm (dotted purple line), also both the profile of the in-focus image (continuous black line), and outof-focus image (continuous green line). 
Also we present profiles of the restored images of a selected region in the image (marked in red), they appear in Figure 3 for the objective 4X(4.1); in Figure 5 are the profiles for the objective $10 \mathrm{X}(0.3)$, and in Figure 7 the profiles for the objective $20 X(0.5)$ are shown.

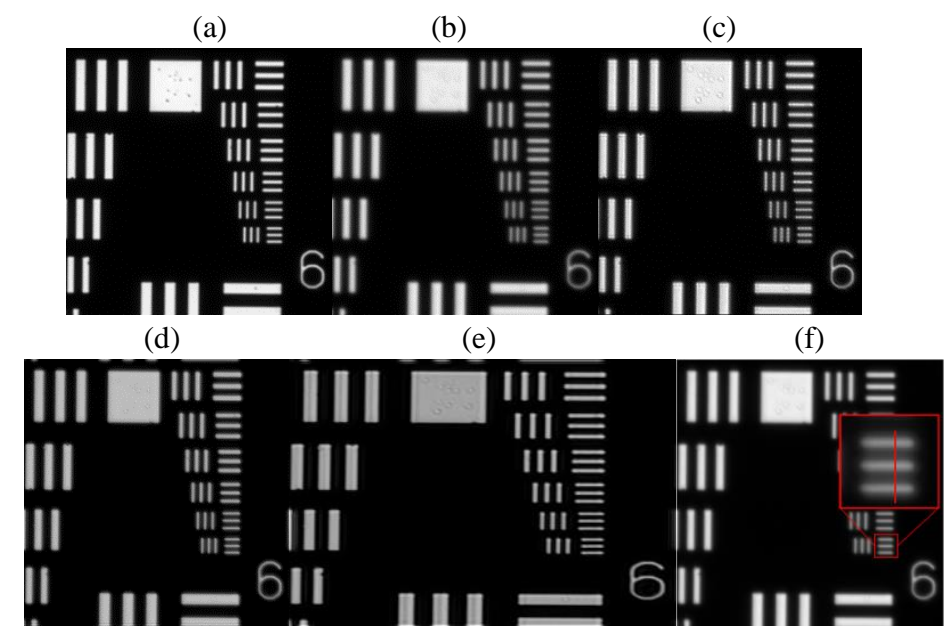

Fig. 4. a) in-focus image of the resolution test target detected with a microscopy objective $10 \mathrm{X}(0.3)$; b) defocused image of the object which amount of defocus is $\omega_{2,0}=0.585 ; \mathrm{c}$ ) restoration of the defocused using a simulated ipsf; d) restoration with the Blind deconvolution algorithm, with 100 iterations; e) restoration with our proposed optical-digital technique; f) this image is showing the transverse section that is used for the profiles plotted in the figure 5.

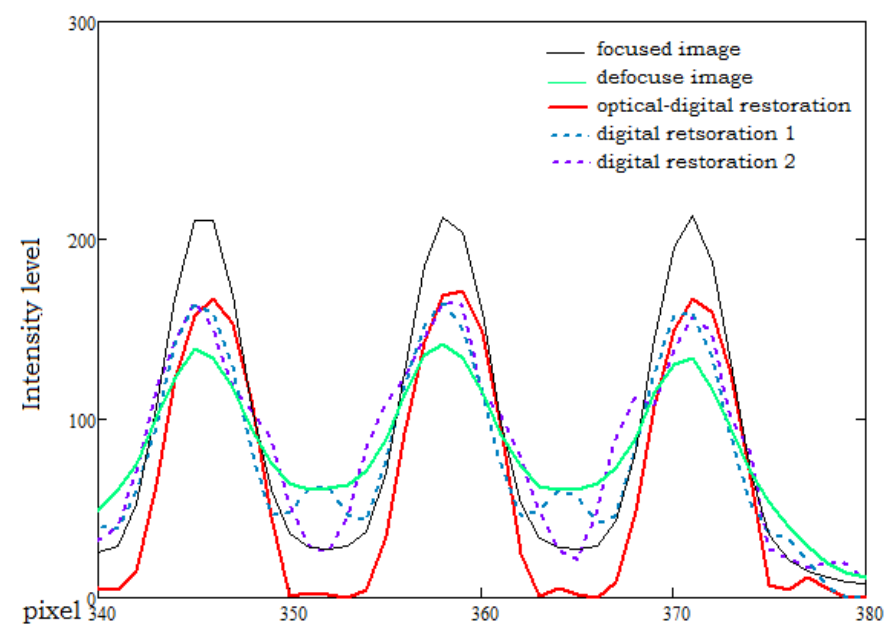

Fig. 5. Intensity profiles in the indicated region in Figure 4(f). Optical-digital technique (continuous red line), using a simulated ipsf (dotted blue line), the Blind deconvolution algorithm (dotted purple line), also both the profile of the in-focus image (continuous black line), and outof-focus image (continuous green line). 


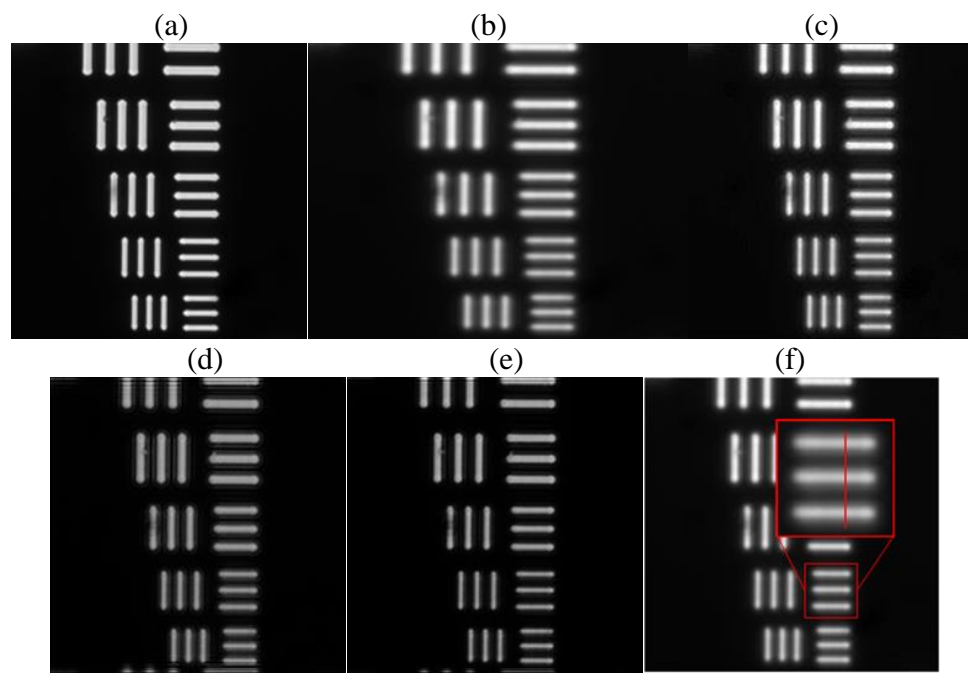

Fig. 6. a) in-focus image of the resolution test target detected with a microscopy objective $20 \mathrm{X}(0.5) ; \mathrm{b})$ defocused image of the object which amount of defocus is $\omega_{2,0}=0.813 ; \mathrm{c}$ ) restoration of the defocused using a simulated ipsf; d) restoration with the Blind deconvolution algorithm, with 100 iterations; e) restoration with our proposed optical-digital technique; f) this image is showing the transverse section that is used for the profiles plotted in the figure 7.

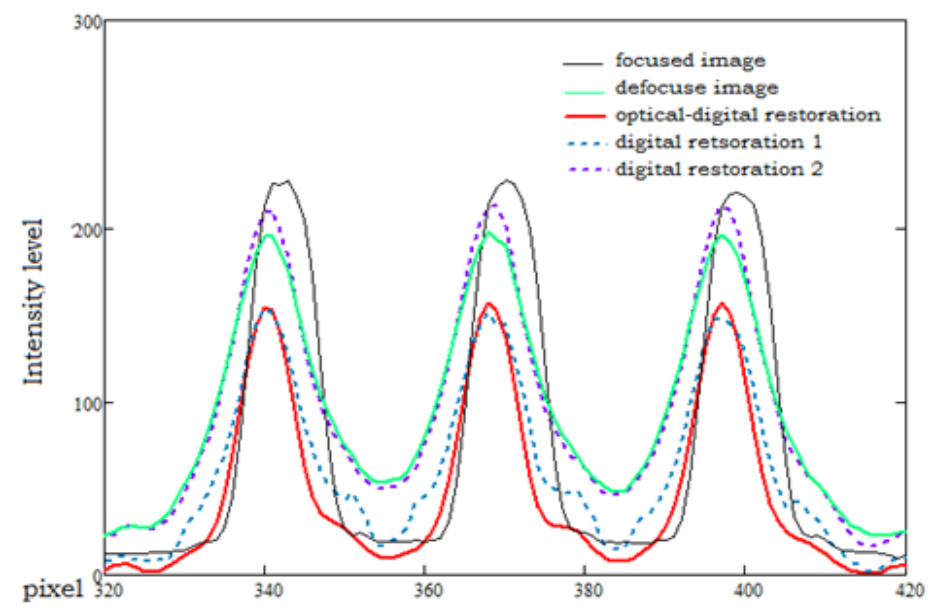

Fig. 7. Intensity profiles in the indicated region in Figure 6(f). Optical-digital technique (continuous red line), using a simulated ipsf (dotted blue line), the Blind deconvolution algorithm (dotted purple line), also both the profile of the in-focus image (continuous black line), and outof-focus image (continuous green line).

\section{Conclusions}

Although, for a rigorous comparison is necessary a quantitative evaluation of the restoration techniques, for the moment we have made only a visual evaluation. 
However with a simple visual inspection of the restoration, is easily appreciated the differences between our optical-digital method with the only digital. The advantages of the optical-digital method over the other two are evident in the three groups of images. Particularly, in the region of the image containing high frequencies, where the edges are restored in a fair way. However the contrast in the restoration resulted better for the digital simulated method instead of the other two.

After observing and comparing all the restored images, we can conclude that the method presented here works for restoring images detected out-of focus for the three different numerical apertures used in the experiment, and provides some advantages over the other two methods. Nevertheless a more exhaustive study is necessary for a complete characterization of our technique.

\section{References}

1. Weinstein. W.: Image of incoherent illuminated bright and opaque disks. J. Opt. Soc. Am., 45(12), pp. 1006-1007 (1955)

2. Mejía-Romero, S., Berriel-Valdos, L. R., Aguilar, J. F., Orlando, I. J., Landgrave, J. E. A.: Discrete Wigner distribution function applied on images of quasi-point source objects in coherent illumination. In: SPIE Proceedings 8011, 22nd Congress of the International Commission for Optics: Light for the Development of the World, Puebla Mexico (2011)

3. Ortiz-Sosa, R., Berriel-Valdos, L. R., Aguilar, J. F., Carranza-Gallardo, J., OrlandoGuerrero, I. J., Mejía-Romero, S.: Optical-digital restoration of out-of-focus color images detected in microscopy. In: SPIE Proceedings 8011, 22nd Congress of the International Commission for Optics: Light for the Development of the World, Puebla Mexico. (November 2011),

4. Gonzales, R. C., Goods, R. E.: Digital image processing. 3rd. Ed., New Jersey, Pearson Prentice Hall (2008)

5. Raveh, I., Mendlovic, D., Zalevsky, Z., Lohmann, A. W.: Digital method for defocus corrections: experimental results. Optical Engineering, 38(10), pp. 1620-1626 (1999)

6. Berriel, L. R., Bescós, J., Santisteban, A.: Image restoration for a defocused optical system. Applied Optics, 22(18), pp. 2772-2780 (1983)

7. Fish, D. A., Brinicombe, A. M., Pike, E. R.: Blind deconvolution by means of the Richardson-Lucy algorithm. J. Opt. Soc. Am., 12(1), pp. 58-65 (1995)

8. Hopkins, H. H.: Wave theory of aberrations. New York: Oxford Univ. Press (1950)

9. Hopkins, H. H.: The Frequency Response of a Defocused Optical System. Proceedings of the Royal Society of London, Series A, Mathematical and Physical Sciences, 231(1184), pp. 91-103 (1955)

10. Goodman, J. W.: Introduction to Fourier Optics. 2nd. Ed., New York, McGraw-Hill (1968) 